\title{
16. SEDIMENT FLUXES IN THE GULF OF ALASKA: PALEOCEANOGRAPHIC RECORD FROM SITE 887 ON THE PATTON-MURRAY SEAMOUNT PLATFORM ${ }^{1}$
}

\author{
David K. Rea ${ }^{2}$ and Hilde Snoeckx ${ }^{2}$
}

\begin{abstract}
The mass accumulation rate record of the major sedimentary components at Ocean Drilling Program Site 887 permits a reconstruction of the paleoceanography and paleoclimatology of the Gulf of Alaska region during the last 18 m.y. Opal fluxes, presumed to reflect sea-surface biological productivity, increase slowly from about 12-6 Ma, are high from 6-2.6 Ma, and peak again in the Quaternary. The 6-2.6 Ma flux maximum is coincident with a time of globally enhanced biological productivity. The Quaternary opal flux maximum at $0.8-0.2 \mathrm{Ma}$ is not seen elsewhere in the North Pacific and probably reflects an intensification of the upwelling associated with the Alaska Gyre. Fluxes of terrigenous sediment permit estimation of the timing of the uplift of the coast ranges of Alaska, the latest Miocene initiation of glaciation there, and when those glaciers reached sea level. These events are linked with the onset of deep-sea fan formation. The highest rates of terrigenous sediment input occurred during the Pliocene-Pleistocene, when the flux of both coarse $(>63 \mathrm{~mm})$ and fine terrigenous grains respond to glaciations in the Gulf of Alaska region.
\end{abstract}

\section{INTRODUCTION}

Site $887\left(54^{\circ} 21.9^{\prime} \mathrm{N}, 148^{\circ} 26.8^{\prime} \mathrm{W}, 3633 \mathrm{~m}\right)$ anchors the eastern end of the North Pacific transect drilled by Ocean Drilling Program (ODP) Leg 145 (Fig. 1). This site is located on the eastern end of the platform level of the Patton-Murray Seamount group (Fig. 2). Many seamount groups consist of multiple peaks atop a common base or platform which may stand as much as $1 \mathrm{~km}$ above the original sea floor. The strategy of drilling the seamount platforms in the Gulf of Alaska was developed at the Seattle INPAC Workshop as a means of avoiding the thick turbidite deposits that blanket the deep-sea floor in the northeast Pacific and recovering a more nearly pure pelagic and hemipelagic record. The workshop report (INPAC, 1985) proposed that ODP take advantage of these "pelagic windows" to recover appropriate sedimentary sections in search of paleoceanographic and paleoclimatic information.

Among the several paleoceanographic and paleoclimatic objectives at Site 887 was the determination of the fluxes of the major sediment components (i.e., opaline silica, calcium carbonate, and terrigenous sediment) to the Gulf of Alaska. We presume that the silica flux data provide a record of the general biological productivity of the Alaska Gyre region, and that the terrigenous flux data reflect some combination of mountain uplift and climate change in southern and southeastern Alaska. Terrigenous sediments at Site 887 are a combination of eolian dust, hemipelagic silts and clays, and ice-rafted debris (IRD). The presence of IRD is judged on the basis of its larger size, generally coarser than some arbitrary diameter; we use 63 $\mathrm{mm}$ in this paper. Site 887 was specifically chosen to lie shallower than the turbidite abyssal plains of the Gulf of Alaska and no turbidite layers were found in the cores. It has been shown elsewhere that the mass accumulation rate history of hemipelagic and turbidite sediments in the same basin have the same temporal pattern (Rea, 1992,

Rea, D.K., Basov, I.A., Scholl, D.W., and Allan, J.F. (Eds.), 1995. Proc. ODP, Sci. Results, 145: College Station, TX (Ocean Drilling Program).

${ }^{2}$ Department of Geological Sciences, University of Michigan, Ann Arbor, MI 48109-1063, U.S.A.
1993). A small portion of the terrigenous grains will consist of eolian dust transported in the westerly winds from Asia. The mass accumulation rates of dust are generally much lower than those of hemipelagic muds (Rea, 1993), so dust is presumed to be a minor component at Site 887 , which is dominated by the hemipelagic (fluvial) runoff from southern Alaska.

\section{Sediments and Analyses}

Although minor amounts of carbonate do occur in the section, the sediments at Site 887 are essentially a two-component mix of siliceous ooze and terrigenous silts and clays. In the upper $90 \mathrm{~m}$, sediments are about $35 \%-50 \%$ biogenic silica; this value increases rapidly downcore at about $110 \mathrm{~m}$ below sea floor (mbsf) to values of $80 \%-90 \%$ siliceous ooze in the older materials (Fig. 3). Calcite is an important component of the sediment in two intervals: 95-112 and 200-260 mbsf (Fig. 3). The site bottomed at $280 \mathrm{mbsf}$ in the basalt of the Patton-Murray Seamount platform.

We sampled Site 887 at regular depth intervals, commonly every three meters. The resulting 73 samples were returned to the laboratory where they were freeze dried and subjected to a mineral extraction procedure that isolates the terrigenous component. This procedure is described by Rea and Janecek (1981), with modifications suggested by Clemens and Prell (1990) and by Hovan (1995). The sequential extraction procedure removes carbonates with acetic acid, opal with $\mathrm{NaOH}$, and oxides and hydroxides with a strong reducing agent. Zeolites are removed by this procedure but volcanic ash survives. We avoided sampling ash layers, but minor amounts of diffuse ash may be present in some of our samples. The $\mathrm{NaOH}$ treatment, necessary for opal removal in these silica-rich sediments, is more harsh than the $\mathrm{Na}_{2} \mathrm{CO}_{3}$ treatment we use in low-silica sediments, and it may remove submicron, neoformational smectites along with the opal. We have no indication that the well-crystallized clay minerals coming from the continent are affected by the extraction procedure. The results of the extraction process yield the weight percent of terrigenous materials in each sample (Fig. 4). Based on duplicate extractions, these values are accurate to $\pm 5 \%$. The coarse component, as determined by screening at $63 \mathrm{~mm}$, is tabulated separately but included in the totals for 


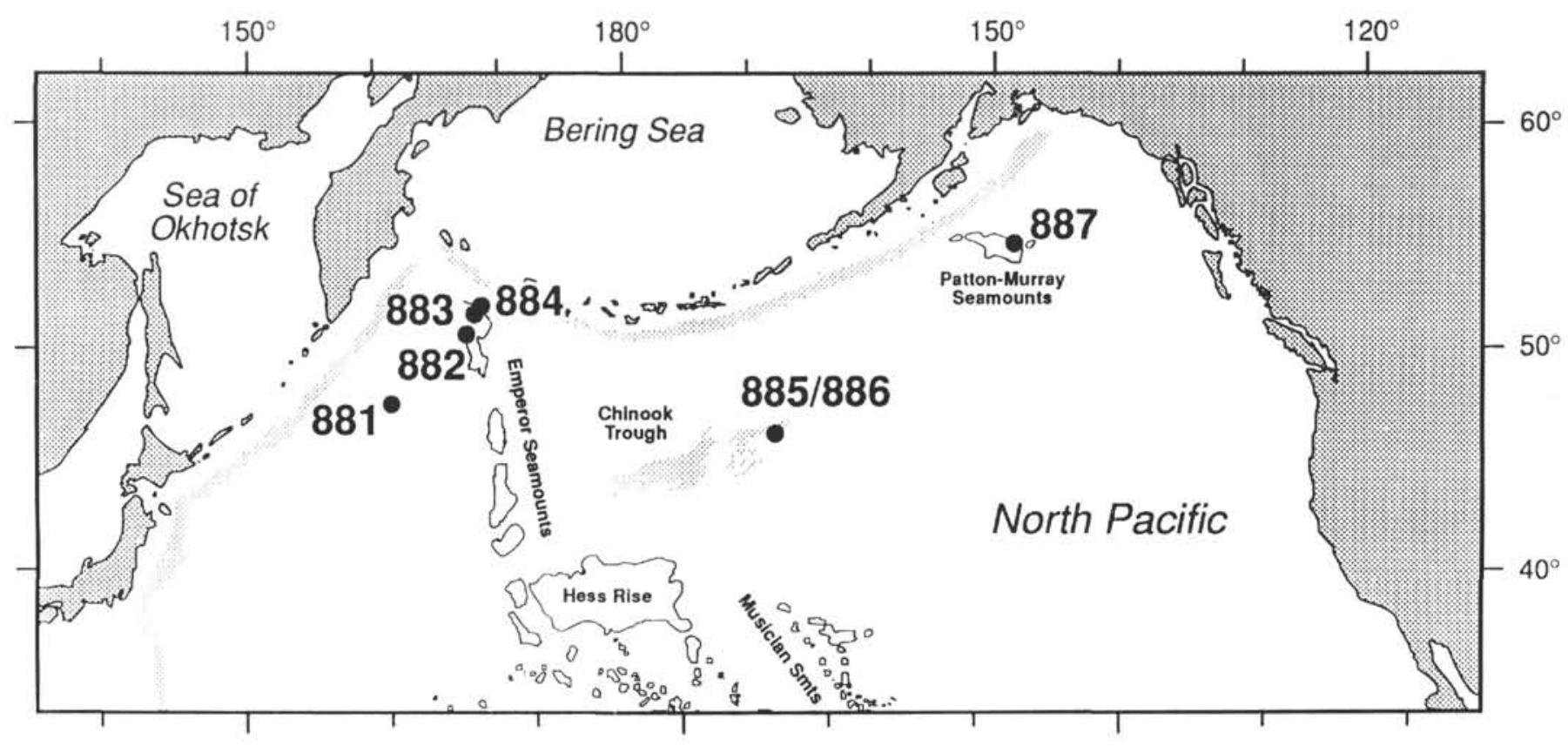

Figure 1. Map of the North Pacific Ocean showing Leg 145 sites.

mass-flux calculations. We then determine opal percentages by difference, allowing for the amount of calcium carbonate reported in parts of the section determined by interpolation between shipboard measurements (Rea, Basov, Janecek, Palmer-Julson, et al., 1993).

Age control for this work is taken from the magnetic reversal stratigraphy determined on board the JOIDES Resolution, a stratigraphy that is both complete and continuous throughout the length of the core (Rea, Basov, Janecek, Palmer-Julson, et al., 1993). From this stratigraphy and the magnetic reversal time scale of Cande and Kent (1992) we constructed both age-depth and linear sedimentation rate relations (Fig. 5). The linear sedimentation rates are very high for the past $0.75 \mathrm{~m} . \mathrm{y}$. (about $6 \mathrm{~cm} / \mathrm{k} . \mathrm{y}$.), decline to $1.3 \mathrm{~cm} / \mathrm{k}$.y. at about 2.0 $\mathrm{Ma}$, and then stay generally at $2-3 \mathrm{~cm} / \mathrm{ky}$ down to $6.0 \mathrm{Ma}$. Sediments older than $6.0 \mathrm{Ma}$ were deposited at normal pelagic rates of rates of 0.5 to $1.5 \mathrm{~cm} / \mathrm{k} . \mathrm{y}$.

To quantify sediment flux, it is necessary to determine the mass accumulation rate of each sedimentary component. The mass accumulation rate (MAR) is the product of the linear sedimentation rate (LSR) and the dry bulk density (DBD) of the sediment such that

$$
\operatorname{MAR}\left[\mathrm{g} /\left(\mathrm{cm}^{2} \mathrm{k} \cdot \mathrm{y} \cdot\right)\right]=\operatorname{LSR}(\mathrm{cm} / \mathrm{k} \cdot \mathrm{y} .) \times \operatorname{DBD}\left(\mathrm{g} / \mathrm{cm}^{3}\right) .
$$

For each sedimentary component MAR is determined by

$$
\mathrm{wt} \% \text { component } \times \text { MARtotal }=\text { MARcomponent. }
$$

The DBD values can be taken from the shipboard physical properties data (Rea, Basov, Janecek, Palmer-Julson, et al., 1993) or determined by water loss from the samples upon freeze drying. All data are shown in Table 1.

\section{Sedimentation Record in the Gulf of Alaska}

\section{Opal}

Documentation of the silica shift, the Miocene enhancement of silica deposition in the North Pacific, was a major paleoceanographic objective for Leg 145. Silica fluxes are taken to be a function of the biological productivity supported by diffuse upwelling, as deep ocean waters reach the end of their circuit in the North Pacific. Site 887 is located along the northeastern margin of the modern upwelling regime associated with the center of the Alaska Gyre (Dodimead et al., 1963) and serves as a monitor of that upwelling.

Biogenic silica fluxes in the Gulf of Alaska are generally very high (Fig.6). The lowest Site 887 values of 200 to $250 \mathrm{mg} /\left(\mathrm{cm}^{2} \mathrm{k} . \mathrm{y}\right.$.) that pertain from 18 to $12.5 \mathrm{Ma}$ are twice those of the modern equatorial Pacific high-productivity zone (Rea et al., 1991). Opal MAR values double to about $500 \mathrm{mg} /\left(\mathrm{cm}^{2} \mathrm{k} . \mathrm{y}\right.$.) beginning at $12.5 \mathrm{Ma}$ and persist through most of the late Miocene. The first major change is a two- to threefold increase in silica fluxes that occurs at $6 \mathrm{Ma}$. Flux values remain high, in the 1000 to $1500 \mathrm{mg} /\left(\mathrm{cm}^{2} \mathrm{k} . \mathrm{y}\right.$.) range, until about 2.6 Ma, with an intervening low centered at 3.3 Ma. These times of high silica flux (Fig. 6) match in time those found throughout the North Pacific transect and denotes the latest Miocene to early late Pliocene productivity maximum. This is the expression in the Gulf of Alaska of the "Diatom Dump" seen at Detroit Seamount Sites 882, 883 and 884 (Rea, Basov, Janecek, Palmer-Julson, et al., 1993), and in the central North Pacific at Sites 885/886 (Snoeckx et al., this volume).

Following the Diatom Dump interval, there is a 0.7-m.y.-long period of decreased opal deposition which continued until about 1.8 Ma. The last $1 \mathrm{~m}$.y. in the Gulf of Alaska are characterized by a pulse of very high opal deposition (Fig. 7) that reaches values in excess $2500 \mathrm{mg} /\left(\mathrm{cm}^{2} \mathrm{k} . \mathrm{y}\right.$.). No equivalent flux episode occurs in the northwest Pacific Pleistocene sediments, so the $0.5-\mathrm{m} . \mathrm{y}$.-long high-productivity episode between about 0.8 and 0.2 Ma may be unique to the Alaska Gyre region.

\section{Calcium carbonate}

The flux of $\mathrm{CaCO}_{3}$ is an intermittent signal at Site 887 (Fig. 8). Two flux maxima of $300-400 \mathrm{mg} /\left(\mathrm{cm}^{2} \mathrm{k} . \mathrm{y}\right.$.) occur in the middle Miocene and a third of similar magnitude corresponds to the early part of the late Miocene, about 9.0-7.5 Ma. The largest flux peak occurs in the mid-Pliocene, between about 3.6 and $2.8 \mathrm{Ma}$, and reaches val- 


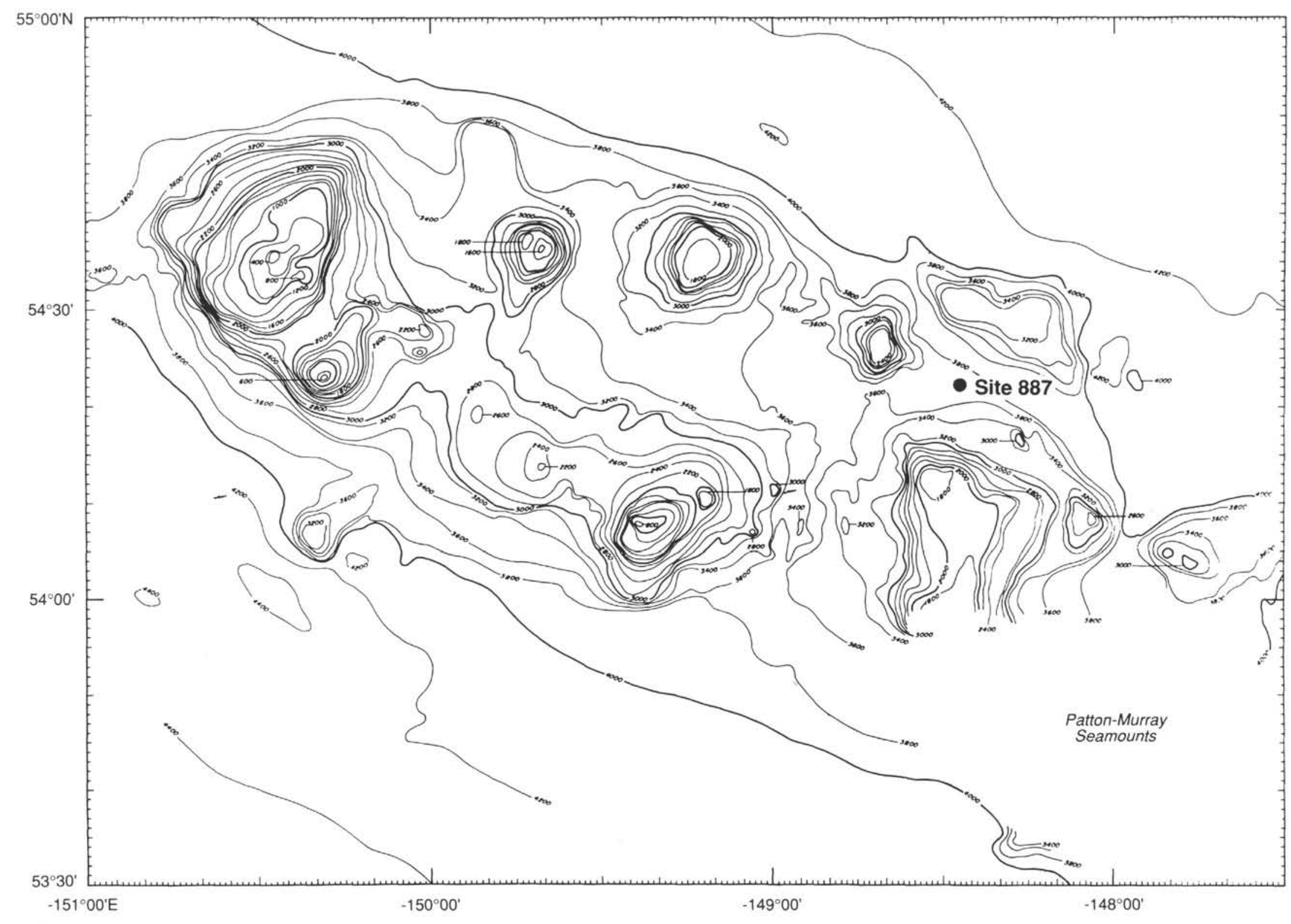

Figure 2. Bathymetric map of the Patton-Murray Seamount group by Carl Brenner of Lamont-Doherty Earth Observatory. Site 887 is at the eastern end of the seamount platform about 10 km north of Murray Seamount. Contour interval is $200 \mathrm{~m}$. 


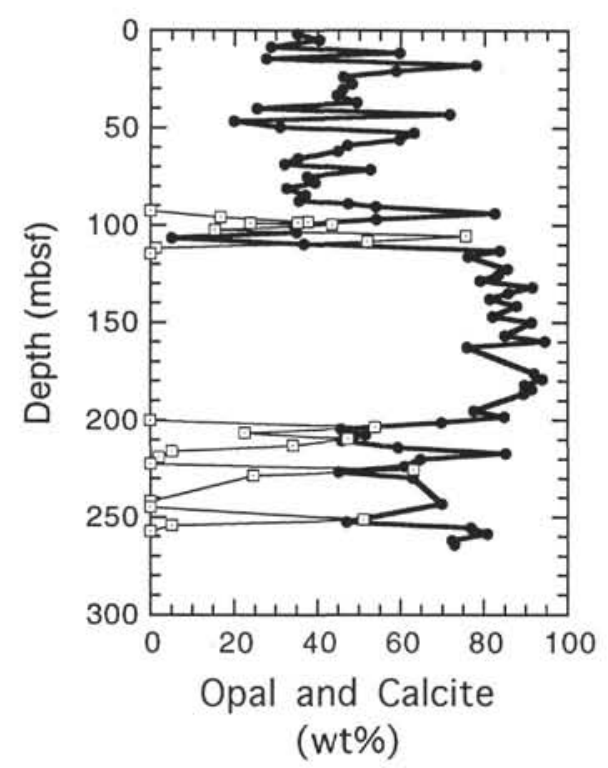

Figure 3.Weight percent of opal (filled circles) and calcite (open squares) in the sediments of Site 887 .

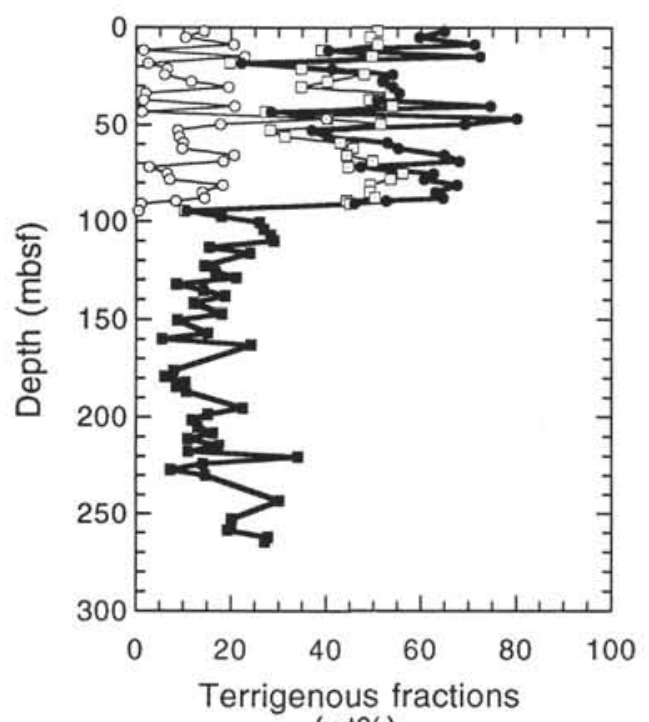

(wt\%)

Figure 4. Weight percent of the terrigenous component of sediments at Site 887. Open circles represent the coarser than $63-\mathrm{mm}$ fraction, open squares represent the mineral component in the fine fraction, and filled symbols represent the total terrigenous component.

ues of $1000 \mathrm{mg} /\left(\mathrm{cm}^{2} \mathrm{k} . \mathrm{y}\right.$.). Sediments of similar age at deeper North Pacific Deep Sea Drilling Project (DSDP) Sites 178 (4218 m; Kulm, von Huene et al., 1973) and 183 (4708 m; Creager, Scholl et al., 1973) are essentially devoid of $\mathrm{CaCO}_{3}$.

\section{Terrigenous sediment}

Throughout the middle Miocene, the MAR of terrigenous materials at Site 887 (Fig. 9) was about $60 \mathrm{mg} /\left(\mathrm{cm}^{2} \mathrm{k} . y\right.$. .), a rather low value for the flux of hemipelagic sediment but several times greater than the middle Miocene eolian flux values of 15 to $20 \mathrm{mg} /\left(\mathrm{cm}^{2} \mathrm{k} . \mathrm{y}\right.$.) recorded at Sites 885/886 (Snoeckx et al., this volume). The first real increase in flux values occurs at $9.9-9.5 \mathrm{Ma}$, rising to values of about

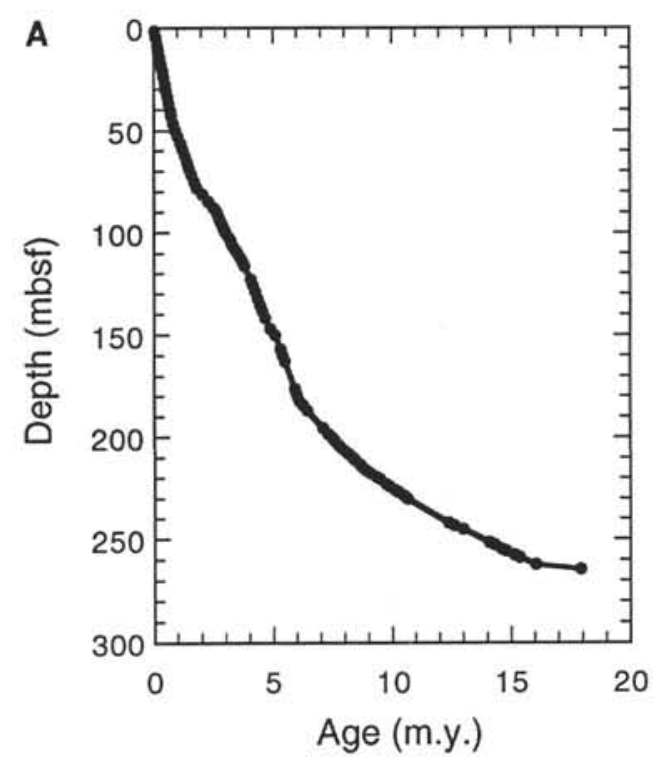

B

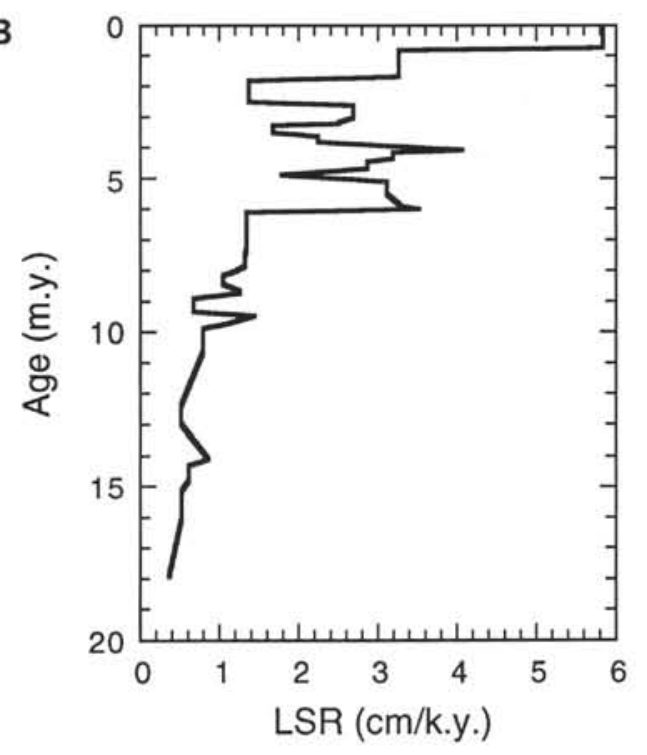

Figure 5. A. Age-depth curve for Site 887 based on magnetic reversal stratigraphy shown in Rea, Basov, Janecek, Palmer-Julson, et al. (1993). B. Linear sedimentation rates calculated from the age-depth relationship.

$100 \mathrm{mg} /\left(\mathrm{cm}^{2} \mathrm{k} . \mathrm{y}.\right)$. Additional terrigenous MAR increases occur at 5.9-5.5 Ma when fluxes double to $200 \mathrm{mg} /\left(\mathrm{cm}^{2} \mathrm{k} . \mathrm{y}\right.$.) and again at about $4.3 \mathrm{Ma}$ when values further increase to $300-400 \mathrm{mg} /\left(\mathrm{cm}^{2} \mathrm{k} . \mathrm{y}\right.$.) (Fig. 9). Two isolated dropstones occur in sediments about 4.2 m.y. old.

During late Pliocene and Pleistocene time, the flux of terrigenous sediment to the Gulf of Alaska increases in three steps to very high input rates (Fig. 10A). Each of these three relative maxima are associated with an increase in the flux of coarser IRD (Fig. 10). Between 2.8 and $2.6 \mathrm{Ma}$, fluxes increased to about $700-800 \mathrm{mg} /\left(\mathrm{cm}^{2} \mathrm{k} . \mathrm{y}\right.$.). A second increase begins $1.8 \mathrm{Ma}$ with a peak at $1.5 \mathrm{Ma}$, and beginning about 1.1 Ma fluxes increased to values of over $2000 \mathrm{mg} /\left(\mathrm{cm}^{2} \mathrm{k} . \mathrm{y}\right.$.), and have remained at 1500 to $2500 \mathrm{mg} /\left(\mathrm{cm}^{2} \mathrm{k} . \mathrm{y}\right.$.) to the top of the core. These high late Pliocene and Pleistocene terrigenous flux values correspond in time to the input of significant amounts of IRD (Fig. 10A; Krissek, this volume). 
Table 1. Sediment percent abundance and mass accumulation rate data for Site 887 .

\begin{tabular}{|c|c|c|c|c|c|c|c|c|c|c|c|}
\hline $\begin{array}{l}\text { Depth } \\
\text { (mbsf) }\end{array}$ & $\begin{array}{l}\text { Age } \\
\text { (Ma) }\end{array}$ & $\begin{array}{l}\text { Terrig } \\
(\mathrm{wt} \%) \\
<63 \mu \mathrm{m}\end{array}$ & $\begin{array}{c}\text { Terrig } \\
(w t \%) \\
>63 \mu \mathrm{m}\end{array}$ & $\begin{array}{l}\text { Total } \\
\text { Terrig } \\
(w t \%)\end{array}$ & $\begin{array}{c}\mathrm{CaCO}_{3} \\
(\mathrm{wt} \%) \\
\text { interpolated }\end{array}$ & $\begin{array}{r}\mathrm{CaCO}_{3} \\
(\mathrm{wt} \%)\end{array}$ & $\begin{array}{c}\text { Opal } \\
\text { (wt\%) }\end{array}$ & $\begin{array}{c}\text { LSR } \\
(\mathrm{cm} / \mathrm{k} . \mathrm{y} .)\end{array}$ & $\begin{array}{c}\text { DBD } \\
\left(\mathrm{g} / \mathrm{cm}^{3}\right)\end{array}$ & $\begin{array}{c}\text { Total Terrig } \\
\text { MAR } \\
\text { (mg/cm²/k.y.) }\end{array}$ & $\begin{array}{c}\text { Terrig } \\
\text { MAR } \\
\text { (3-pt smooth) }\end{array}$ \\
\hline 1.90 & 0.03 & 50.7 & 14.2 & 64.9 & & & 35.1 & 5.84 & 0.65 & 2463 & 2106 \\
\hline 4.90 & 0.08 & 49.2 & 10.4 & 59.6 & & & 40.4 & 5.84 & 0.40 & 1391 & 1623 \\
\hline 8.60 & 0.15 & 50.8 & 20.5 & 71.2 & & & 28.8 & 5.84 & 0.30 & 1248 & 1414 \\
\hline 11.60 & 0.20 & 38,9 & 1.5 & 40.3 & & & 59.7 & 5.84 & 0.75 & 1767 & 2199 \\
\hline 14.60 & 0.25 & 49.5 & 22.8 & 72.3 & & & 27.7 & 5.84 & 0.95 & 4013 & 2690 \\
\hline 18.10 & 0.31 & 19.6 & 2.5 & 22.1 & & & 77.9 & 5.84 & 0.75 & 968 & 1908 \\
\hline 21.10 & 0.36 & 34.6 & 6.5 & 41.2 & & & 58.8 & 5.84 & 0.70 & 1682 & 1870 \\
\hline 24.10 & 0.41 & 47.9 & 6.0 & 53.9 & & & 46.1 & 5.84 & 1.00 & 3147 & 2371 \\
\hline 27.60 & 0.47 & 40.2 & 11.5 & 51.7 & & & 48.3 & 5.84 & 0.50 & 1510 & 2369 \\
\hline 30.60 & 0.52 & 34.6 & 19.4 & 54.0 & & & 46.0 & 5.84 & 1.05 & 3310 & 2720 \\
\hline 33.60 & 0.58 & 53.5 & 1.9 & 55.4 & & & 44.6 & 5.84 & 0.85 & 2748 & 2718 \\
\hline 37.10 & 0.64 & 49.0 & 1.5 & 50.6 & & & 49.4 & 5.84 & 0.70 & 2067 & 2264 \\
\hline 40.10 & 0.69 & 53.9 & 20.6 & 74.5 & & & 25.5 & 5.84 & 0.50 & 2175 & 1831 \\
\hline 43.10 & 0.74 & 27.1 & 1.2 & 28.3 & & & 71.7 & 5.84 & 0.55 & 908 & 1715 \\
\hline 46.60 & 0.81 & 40.1 & 40.0 & 80.1 & & & 19.9 & 3.26 & 1.10 & 2869 & 2252 \\
\hline 49.61 & 0.90 & 51.4 & 17.6 & 69.0 & & & 31.0 & 3.26 & 1.05 & 2361 & 2003 \\
\hline 52.60 & 1.00 & 28.1 & 8.8 & 36.8 & & & 63.2 & 3.26 & 0.35 & 420 & 997 \\
\hline 56.09 & 1.10 & 31.2 & 9.0 & 40.3 & & & 59.7 & 3.26 & 0.60 & 787 & 735 \\
\hline 59.09 & 1.20 & 42.9 & 9.9 & 52.8 & & & 47.2 & 3.26 & 0.55 & 945 & 1028 \\
\hline 62.09 & 1.29 & 45.6 & 9.6 & 55.1 & & & 44.9 & 3.26 & 0.80 & 1437 & 1324 \\
\hline 65.60 & 1.40 & 44.2 & 20.6 & 64.8 & & & 35.2 & 3.26 & 0.70 & 1477 & 1679 \\
\hline 68.60 & 1.49 & 49.7 & 18.3 & 68.0 & & & 32.0 & 3.26 & 1.05 & 2324 & 1839 \\
\hline 71.60 & 1.58 & 44.6 & 2.7 & 47.2 & & & 52.8 & 3.26 & 0.80 & 1231 & 1655 \\
\hline 75.08 & 1.69 & 56.0 & 6.5 & 62.6 & & & 37.4 & 3.26 & 0.90 & 1834 & 1432 \\
\hline 78.08 & 1.81 & 53.6 & 7.0 & 60.6 & & & 39.4 & 1.37 & 1.00 & 828 & 1103 \\
\hline 81.10 & 2.03 & 49.3 & 18.2 & 67.5 & & & 32.5 & 1.37 & 1.00 & 923 & 809 \\
\hline 84.60 & 2.29 & 49.1 & 13.8 & 62.9 & & & 37.1 & 1.37 & 0.65 & 559 & 731 \\
\hline 87.60 & 2.50 & 50.2 & 14.4 & 64.5 & & & 35.5 & 1.37 & 1.00 & 883 & 882 \\
\hline 89.10 & 2.61 & 44.2 & 8.4 & 52.6 & & & 47.4 & 2.69 & 0.85 & 1201 & 1022 \\
\hline 90.60 & 2.66 & 44.9 & 1.0 & 45.9 & & & 54.1 & 2.69 & 0.65 & 802 & 735 \\
\hline 92.88 & 2.75 & & & & & 0.0 & & 2.69 & 0.46 & & \\
\hline 94.10 & 2.79 & 10.1 & 0.5 & 10.6 & 6.9 & & 82.6 & 2.69 & 0.48 & 137 & 342 \\
\hline 95.87 & 2.86 & & & & & 16.8 & & 2.69 & 0.62 & 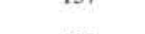 & 20 \\
\hline 97.10 & 2.91 & 17.9 & & 17.9 & 28.0 & & 54.1 & 2.69 & 0.61 & 293 & 268 \\
\hline 98.17 & 2.94 & & & & & 37.7 & & 2.69 & 0.75 & & \\
\hline 98.82 & 2.97 & & & & & 35.3 & & 2.69 & 0.90 & & \\
\hline 98.91 & 2.97 & & & & & 23.9 & & 2.69 & 0.93 & & \\
\hline 99.75 & 3.00 & & & & & 43.5 & & 2.69 & 0.90 & & \\
\hline 100.10 & 3.02 & 25.9 & & 25.9 & 39.8 & & 34.3 & 2.69 & 0.50 & 348 & 341 \\
\hline 102.41 & 3.17 & & & & & 15.4 & & 2.50 & 0.50 & 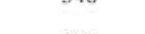 & Sin \\
\hline 103.60 & 3.22 & 26.8 & & 26.8 & 38.2 & & 35.0 & 2.50 & 0.56 & 375 & 351 \\
\hline 105.55 & 3.27 & & & & & 75.6 & & 1.67 & 0.93 & & \\
\hline 106.60 & 3.33 & 28.2 & & 28.2 & 66.8 & & 5.0 & 1.67 & 0.65 & 305 & 313 \\
\hline 108.36 & 3,43 & & & & & 52.1 & & 1.67 & 0.83 & & \\
\hline 109.60 & 3.51 & 29.0 & & 29.0 & 34.4 & & 36.7 & 1.67 & 0.55 & 266 & 252 \\
\hline 111.91 & 3.62 & & & & & 1.4 & & 2.24 & 0.53 & & \\
\hline 113.10 & 3.68 & 15.4 & & 15.4 & 0.8 & & 83.8 & 2.24 & 0.50 & 172 & 225 \\
\hline 114.88 & 3.76 & & & & & 0.0 & & 2.24 & 0.53 & & \\
\hline 116.10 & 3.81 & 23.9 & & 23.9 & & & 76.1 & 2.24 & 0.54 & 289 & 259 \\
\hline 122.60 & 4.07 & 14.4 & & 14.4 & & & 85.6 & 4.06 & 0.49 & 287 & 282 \\
\hline 125.60 & 4.15 & 16.6 & & 16.6 & & & 83.4 & 3.19 & 0.50 & 265 & 281 \\
\hline 128.60 & 4.24 & 21.0 & & 21.0 & & & 79.0 & 3.19 & 0.46 & 308 & 248 \\
\hline 132.10 & 4.35 & 8.4 & & 8.4 & & & 91.6 & 3.19 & 0.41 & 110 & 178 \\
\hline 135.10 & 4.45 & 14.2 & & 14.2 & & & 85.8 & 2.86 & 0.45 & 183 & 182 \\
\hline 138.04 & 4.55 & 18.6 & & 18.6 & & & 81.4 & 2.86 & 0.47 & 251 & 209 \\
\hline 141.60 & 4.67 & 12.2 & & 12.2 & & & 87.8 & 2.86 & 0.44 & 153 & 180 \\
\hline 147.00 & 4.88 & 18.0 & & 18.0 & & & 82.0 & 1.77 & 0.51 & 162 & 148 \\
\hline 150.00 & 5.09 & 8.6 & & 8.6 & & & 91.4 & 3.11 & 0.42 & 112 & 147 \\
\hline 156.70 & 5.30 & 15.0 & & 15.0 & & & 85.0 & 3.11 & 0.43 & 201 & 148 \\
\hline 159.70 & 5.40 & 5.4 & & 5.4 & & & 94.6 & 3.11 & 0.47 & 79 & 172 \\
\hline 162.70 & 5.50 & 24.0 & & 24.0 & & & 76.0 & 3.11 & 0.44 & 329 & 217 \\
\hline 176.00 & 5.91 & 8.0 & & 8.0 & & & 92.0 & 3.30 & 0.50 & 132 & 183 \\
\hline 179.00 & 6.00 & 6.0 & & 6.0 & & & 94.0 & 3.52 & 0.66 & 139 & 122 \\
\hline 182.00 & 6.10 & 10.3 & & 10.3 & & & 89.7 & 1.34 & 0.55 & 76 & 86 \\
\hline 184.10 & 6.25 & 8.5 & & 8.5 & & & 91.5 & 1.34 & 0.47 & 53 & 63 \\
\hline 186.60 & 6.44 & 10.5 & & 10.5 & & & 89.5 & 1.34 & 0.50 & 70 & 85 \\
\hline 195.30 & 7.09 & 22.4 & & 22.4 & & & 77.6 & 1.34 & 0.48 & 144 & 115 \\
\hline 198.30 & 7.31 & 15.1 & & 15.1 & & & 84.9 & 1.34 & 0.51 & 103 & 106 \\
\hline 200.12 & 7.49 & & & & & 0.0 & & 1.32 & 0.47 & & \\
\hline 201.30 & 7.58 & 11.8 & & 11.8 & 18.4 & & 69.8 & 1.32 & 0.48 & 75 & 90 \\
\hline 203.58 & 7.75 & & & & & 54.0 & & 1.32 & 0.50 & & \\
\hline 204.80 & 7.84 & 12.9 & & 12.9 & 41.4 & & 45.7 & 1.32 & 0.63 & 107 & 94 \\
\hline 206.62 & 8.02 & & & & & 22.6 & & 1.18 & 0.58 & & \\
\hline 207.80 & 8.14 & 16.2 & & 16.2 & 32.1 & & 51.7 & 1.04 & 0.52 & 88 & 93 \\
\hline 209.70 & 8.33 & & & & & 47.4 & & 1.04 & 0.75 & & \\
\hline 210.80 & 8.43 & 10.8 & & 10.8 & 43.3 & & 46.0 & 1.04 & 0.80 & 90 & 99 \\
\hline 213.20 & 8.64 & & & & & 34.2 & & 1.25 & 0.66 & & \\
\hline 214.30 & 8.73 & 17.4 & & 17.4 & 23.2 & & 59.4 & 1.25 & 0.60 & 130 & 98 \\
\hline 216.11 & 8.88 & & & & & 5.2 & & 0.67 & 0.51 & & \\
\hline 217.30 & 9.06 & 11.0 & & 11.0 & 4.0 & & 85.1 & 0.67 & 0.57 & 42 & 117 \\
\hline 219.11 & 9.33 & & & & & 2.1 & & 0.67 & 0.37 & & \\
\hline 220.30 & 9.47 & 34.0 & & 34.0 & 1.4 & & 64.6 & 1.43 & 0.52 & 253 & 150 \\
\hline 222.61 & 9.73 & & & & & 0.0 & & 1.08 & 0.48 & & \\
\hline 223.80 & 9.87 & 14.1 & & 14.1 & 25.0 & & 60.9 & 0.79 & 0.46 & 51 & 98 \\
\hline 225.61 & 10.10 & & & & & 63.1 & & 0.79 & 0.64 & & \\
\hline 226.80 & 10.25 & 7.2 & & 7.2 & 47.9 & & 45.0 & 0.79 & 0.65 & 37 & 47 \\
\hline
\end{tabular}


Table 1 (continued).

\begin{tabular}{|c|c|c|c|c|c|c|c|c|c|c|c|c|c|c|c|}
\hline $\begin{array}{l}\text { Depth } \\
\text { (mbsf) }\end{array}$ & $\begin{array}{l}\text { Age } \\
(\mathrm{Ma})\end{array}$ & $\begin{array}{c}\text { Terrig } \\
(w t \%) \\
<63 \mu \mathrm{m}\end{array}$ & $\begin{array}{c}\text { Terrig } \\
(\mathrm{wt} \%) \\
>63 \mu \mathrm{m}\end{array}$ & $\begin{array}{l}\text { Total } \\
\text { Terrig } \\
\text { (w1\%) }\end{array}$ & $\begin{array}{c}\mathrm{CaCO}_{3} \\
(\mathrm{wt} \%) \\
\text { interpolated }\end{array}$ & $\begin{array}{l}\mathrm{CaCO}_{3} \\
(\mathrm{w} t \%)\end{array}$ & $\begin{array}{l}\text { Opal } \\
(\mathrm{w} t \%)\end{array}$ & $\begin{array}{c}\text { LSR } \\
(\mathrm{cm} / \mathrm{k} . \mathrm{y} .)\end{array}$ & $\begin{array}{c}\text { DBD } \\
\left(\mathrm{g} / \mathrm{cm}^{3}\right)\end{array}$ & $\begin{array}{c}\text { Total Terrig } \\
\text { MAR } \\
\left(\mathrm{mg} / \mathrm{cm}^{2} / \mathrm{k} . \mathrm{y}\right)\end{array}$ & $\begin{array}{c}\text { Terrig } \\
\text { MAR } \\
\text { (3-pt smooth }\end{array}$ & $\begin{array}{c}\text { Coarse Terrig } \\
\text { MAR } \\
\left(\mathrm{mg} / \mathrm{cm}^{2} / \mathrm{k} . \mathrm{y} .\right)\end{array}$ & $\begin{array}{c}\text { Opal } \\
\text { MAR } \\
\left(\mathrm{mg} / \mathrm{cm}^{2} / \mathrm{k} . \mathrm{y} .\right)\end{array}$ & $\begin{array}{c}\text { Opal } \\
\text { MAR } \\
\text { (3-pt smooth) }\end{array}$ & $\begin{array}{c}\mathrm{CaCO}_{3} \\
\mathrm{MAR} \\
\left(\mathrm{mg} / \mathrm{cm}^{2} / \mathrm{k} . \mathrm{y}\right.\end{array}$ \\
\hline 228.61 & 10.48 & & & & & 24.7 & & 0.79 & 0.55 & & & & & & 107.32 \\
\hline 229.80 & 10.63 & 14.6 & & 14.6 & 22.5 & & 62.9 & 0.79 & 0.54 & 62 & 59 & & 268 & 237 & \\
\hline 241.91 & 12.38 & & & & & 0.0 & & 0.51 & 0.42 & & & & & & 0.00 \\
\hline 243.10 & 12.62 & $\begin{array}{l}2 \\
2\end{array}$ & & 30.0 & 0.0 & & 70.0 & 0.51 & 0.50 & 77 & 75 & & 178 & 156 & \\
\hline 244.91 & 12.97 & & & & & 0.0 & & 0.51 & 0.50 & & & & & & 0.00 \\
\hline 251.41 & 14.10 & & & & & 51.1 & & 0.86 & 0.65 & & & & & & 285.65 \\
\hline 252.60 & 14.30 & 20.1 & & 20.1 & 32.9 & & 47.0 & 0.61 & 0.68 & 83 & 76 & & 195 & 201 & \\
\hline 254.41 & 14.60 & & & & & 5.2 & & 0.61 & 0.51 & & & & & & 16.18 \\
\hline 255.60 & 14.80 & 19.9 & & 19.9 & 3.1 & & 76.9 & 0.61 & 0.50 & 61 & 64 & & 235 & 221 & \\
\hline 257.41 & 15.14 & & & & & 0.0 & & 0.52 & 0.51 & & & & & & 0.00 \\
\hline 258.60 & 15.36 & $\begin{array}{ll}6 & 19.2\end{array}$ & & 19.2 & & & 80.8 & 0.52 & 0.52 & 52 & 58 & & 218 & 215 & \\
\hline 262.10 & 16.04 & $\quad 27.7$ & & 27.7 & & & 72.3 & 0.52 & 0.50 & 72 & 61 & & 188 & 181 & \\
\hline 264.28 & 17.92 & 2. 27.0 & & 27.0 & & & 73.0 & 0.36 & 0.50 & 49 & 56 & & 131 & 150 & \\
\hline
\end{tabular}

Note: $\mathrm{LSR}=$ linear sedimentation rate $\mathrm{DBD}=$ dry bulk density: $\mathrm{MAR}=$ mass accumulation rate.

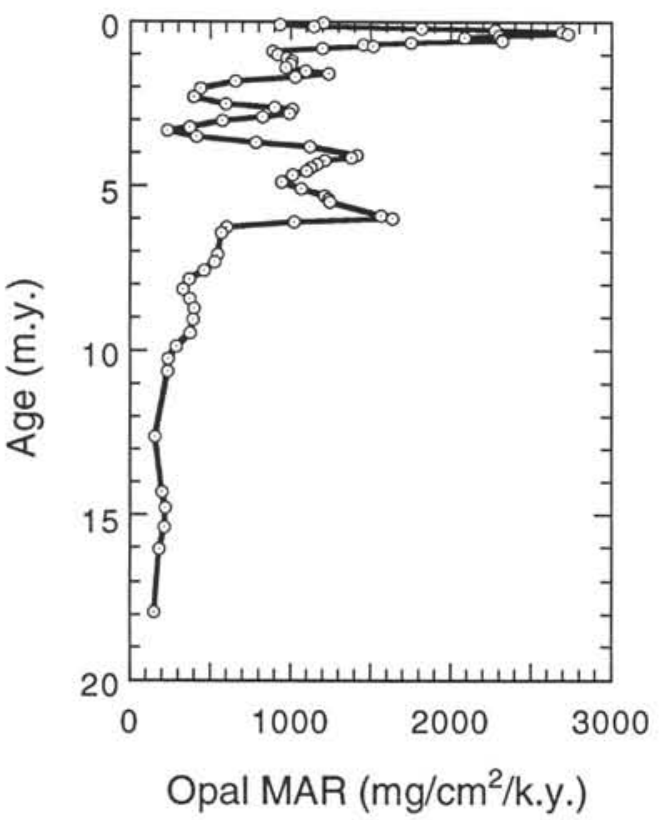

Figure 6. Mass accumulation rate of opal at Site 887 during the past $18 \mathrm{~m} . \mathrm{y}$.

\section{PALEOCEANOGRAPHY AND \\ PALEOCLIMATOLOGY OF THE GULF OF ALASKA REGION \\ North Pacific Paleoceanography}

Opal accumulation rates in the region of the Alaska Gyre increased twice during the Neogene, once starting about 12.5 Ma when MAR values double, and again during the 6.0- to 2.6-Ma period of the late Neogene productivity maxima informally called the Diatom Dump. Each of these presumed productivity enhancements requires an increased supply of nutrients to the photic zone of the northeastern Pacific.

A straightforward interpretation of this regional flux pattern would be to call upon proposed changes in global ocean deep-water circulation. During the Neogene, as the deep-water circulation becomes more like the modern, with important sources in the North Atlantic and in the Weddell Sea, the North Pacific terminus of the deep circulation path would receive an increasing supply of advected nutrients. The upwelling of these nutrient-rich waters, whether "diffuse" or concentrated in the Alaska Gyre region, would then support

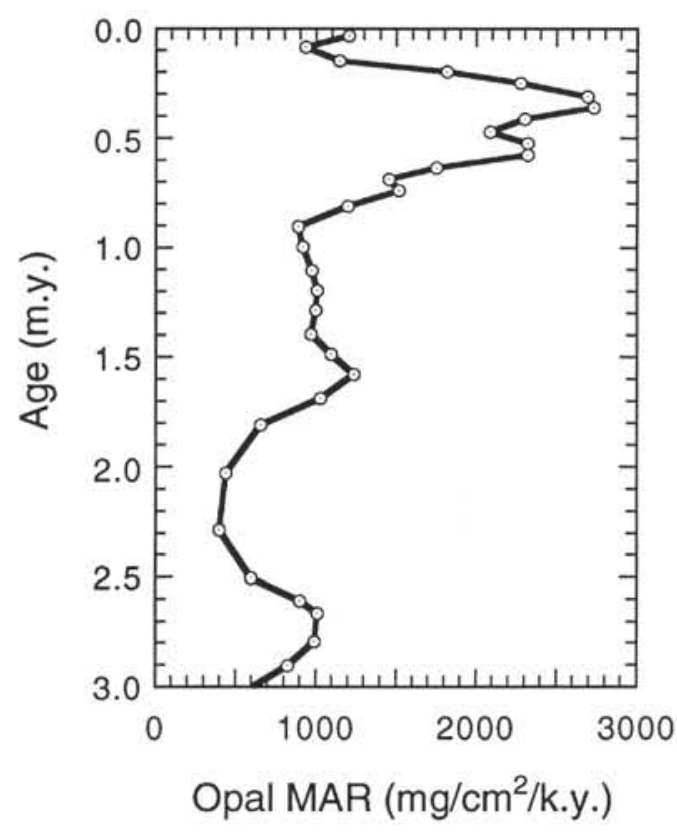

Figure 7. Mass accumulation rate of opal at Site 887 during the past 3 m.y. emphasizing the Quaternary flux maxima.

the Neogene productivity patterns observed. Enhancements in this deep circulation pattern, taken to reflect an increase in Northern (Atlantic) Component Water have been suggested for about $12 \mathrm{Ma}$ (Woodruff and Savin, 1989; Wright et al., 1992); further enhancement may have resulted in a near-modern deep-ocean circulation pattern by $7 \mathrm{Ma}$ (Wright et al., 1991). The onset of Northern Hemisphere glaciation at 2.6 Ma resulted in a suppression of North Atlantic DeepWater formation (Sikes et al., 1991; Raymo et al., 1992), which would sharply reduce the advection of deep-water nutrients to the North Pacific. The timing of these several events matches very well the opal MAR increase at about $12 \mathrm{Ma}$ and the onset and cessation of the Diatom Dump.

Unfortunately, there is a problem with this seemingly satisfactory explanation. The latest Miocene to early late Pliocene productivity maxima appears to be a global phenomenon, and so may not be amenable to a regional or even hemispherical explanation. The results of ODP Leg 138 in the eastern equatorial Pacific (Mayer, Pisias, et al., 1992), Leg 130 in the western equatorial Pacific (Kroenke, Berger, et al., 1990), Leg 115 in the western Indian Ocean (Backman, Duncan, 


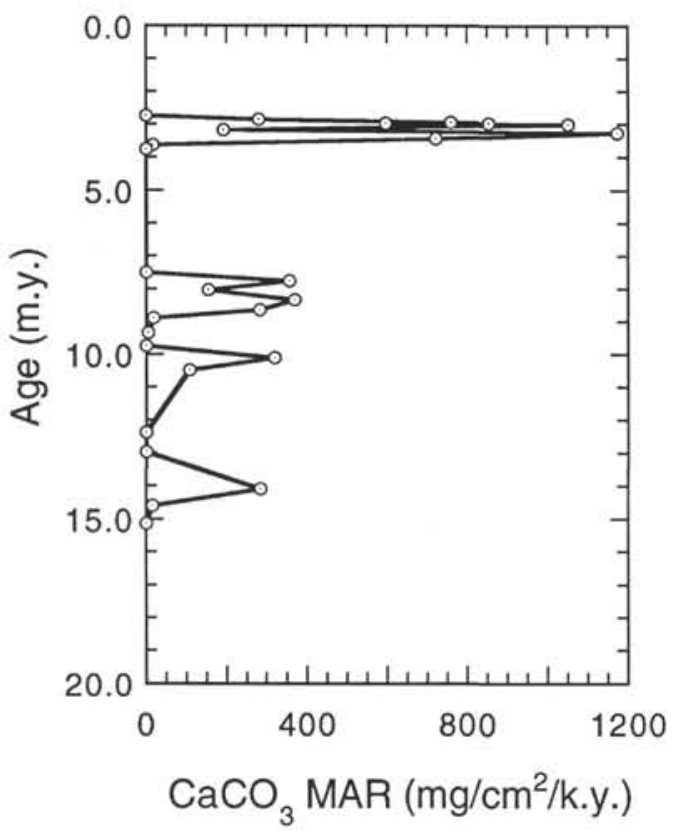

Figure 8. Mass accumulation rate of calcite at Site 887.

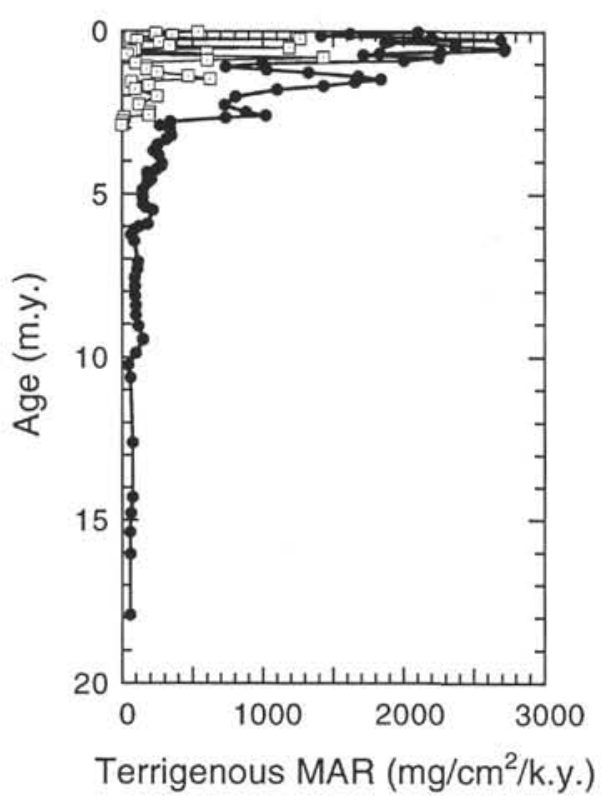

Figure 9. Mass accumulation rate of terrigenous sediment at Site 887. Open squares show the flux of the coarser than $63-\mathrm{mm}$ component; filled circles show 3-point smoothed values of the total terrigenous flux.

et al., 1988; Peterson et al., 1992), and DSDP Leg 74 in the South Atlantic (Moore, Rabinowitz, et al., 1984) all show enhanced MARs of biogenic sediment during this same time interval. This information indicates that there was a worldwide increase in the amount of nutrients coming into the ocean, rather than a redistribution of nutrients within the ocean reservoir. Moore (1993) has suggested that this was caused by a eustatic sea-level rise, with the flooded continental shelves exporting nutrients to the deep sea. Any explanation must account for both the onset of this global productivity maximum at 7-6 $\mathrm{Ma}$, and its cessation about 2.6-3 Ma.
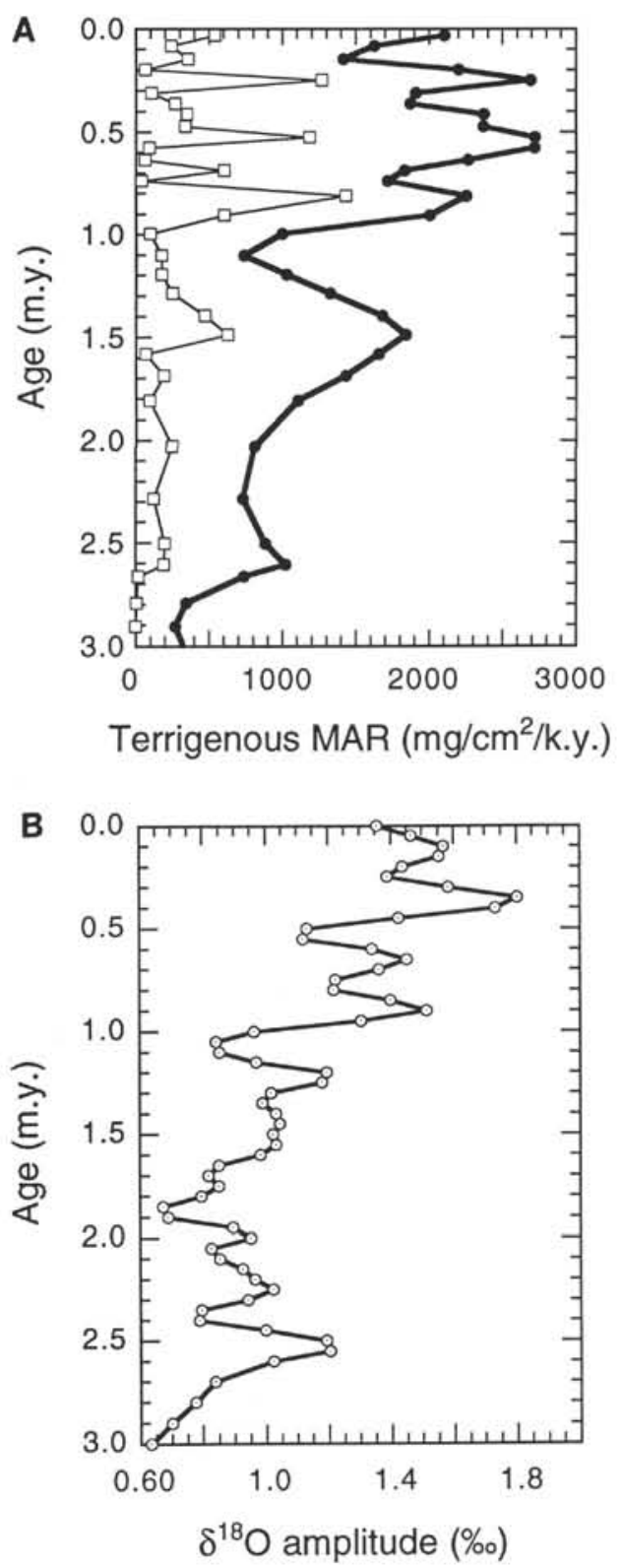

Figure 10. A. Mass accumulation rate of terrigenous sediment at Site 887 showing details of the past $3 \mathrm{~m} . \mathrm{y}$. Open squares show the flux of the coarser than $63-\mathrm{mm}$ component, interpreted as representing ice-rafted debris. Filled circles show total terrigenous component flux values (3-point smoothed). Note the general correspondence between IRD and total flux values. B. Amplitude of the oxygen-isotope record of global change. This plot is a three-point smoothing of the width of an envelope, determined every $50 \mathrm{ka}$, defined by successive maxima and minima of a $\delta^{18} \mathrm{O}$ curve from benthic foraminifera. Original data are from Tiedemann (pers. comm., 1992) for ODP Site 677 in the eastern equatorial Pacific. Note the general correspondence between terrigenous fluxes (A), and the increase in the amplitude of glaciation (B).

The Quaternary opal flux maximum seen at Site 887 is not evident in the other Leg 145 drill sites (Rea, Basov, Janecek, Palmer-Julson, et al., 1993; Haug et al., this volume; Snoeckx et al., this volume). As such, a local to regional explanation may be more appropriate for this young flux maxima. Perhaps there has been increased upwelling associated with the Alaska Gyre that began about $1.8 \mathrm{Ma}$ and intensified at $0.9-0.7 \mathrm{Ma}$. 
Calcite flux peaks record a deepening of the calcite compensation depth, either because of an increased rate of carbonate productivity or a reduced rate of dissolution at depth. The youngest and largest peak corresponds in time to the mid-Pliocene warm interval and so may reflect warmer sea-surface temperatures in the Gulf of Alaska and a corresponding shift to a more calcareous plankton assemblage.

\section{Alaskan Paleoclimatology}

The history of terrigenous sedimentation in the Gulf of Alaska has been a topic of marine geological research for more than $40 \mathrm{yr}(\mathrm{Me}-$ nard, 1953, 1955, 1964; Hays, 1970). DSDP Leg 18 drilling in 1971 resulted in the first reasonable estimate of the timing of various Cenozoic sedimentary events (Kulm, von Huene, et al., 1973). More recently, Stevenson and Embley (1987), von Huene (1989), and Lagoe et al. (1993) have published useful papers that concern the history of terrigenous deposition in the Gulf of Alaska. Sediments of the northeast Pacific are dominated by turbidites that form a series of abyssal plains and deep-sea fans (Fig. 11). The history of the three large deepsea fans, Zodiac, Surveyor, and Baranoff, is not yet fully understood; the record from Site 887 should bear upon the two younger fans, Surveyor and Baranoff, but not upon the more problematic history of the older Zodiac Fan (Stevenson and Embley, 1987; von Huene, 1989). Finally, throughout the time represented by the sedimentary record of Site 887, the Pacific Plate in the Gulf of Alaska has been moving northwest and converging with the Aleutian Trench at a rate of 40 to $50 \mathrm{~km} / \mathrm{m}$.y. (Rea and Duncan, 1986). About $800 \mathrm{~km}$ of convergence have occurred during the 18-m.y.-long record at Site 887, but because of the regional plate geometry, the distance from the shore of southeastern Alaska and British Columbia has not changed very much.

The sediment accumulation record at Site 887 began with 8 m.y. of deposition during the latest early Miocene and entire middle Miocene, characterized by relatively low fluxes of terrigenous sediment. The first increase in terrigenous fluxes occurred in the early portion of the late Miocene, between 9.87 and $9.47 \mathrm{Ma}$. This event was first resolved by the scientists of DSDP Leg 18 who recognized the initial turbidite deposition in the Gulf of Alaska then (Kulm, von Huene, et al., 1973) and associated that event with the uplift of the Coast Ranges in Alaska (see also Lagoe et al., 1993). The beginning of the construction of the Surveyor Fan, which now occupies the northern Gulf of Alaska, was associated with this tectonic and depositional event (Stevenson and Embley, 1987; von Huene, 1989), which we can now date as occurring about $9.7 \mathrm{Ma}$.

At the close of the Miocene, between 5.91 and $5.50 \mathrm{Ma}$, the MAR of terrigenous material in the Gulf of Alaska doubled (Fig. 9). This event occurred at the same general time as the earliest indications of cooling in the North Pacific region, such as the presence of glacioneritic diatoms at DSDP Site 183 and the likely initiation of alpine glaciation in southeastern Alaska (Lagoe et al., 1993). Deposition in the Baranoff Fan, with its sediment sources in British Columbia and Southeast Alaska, probably began at this time (Stevenson and Embley, 1987; von Huene, 1989).

A further increase in terrigenous fluxes, from $<200$ to $>300 \mathrm{mg} /$ ( $\mathrm{cm}^{2} \mathrm{k} . \mathrm{y}$.), occurred between 4.36 and $4.26 \mathrm{Ma}$ (Fig. 9), just prior to the time of deposition of the early Pliocene dropstones recovered in two of the holes at Site 887. This increase and the associated dropstones may be directly comparable with onshore events in the Alaskan coast ranges. The magnetic reversal stratigraphy of the Alaskan glaciomarine Yakataga Formation presented by Lagoe et al. (1993) can be reinterpreted to match the new reversal time scale of Cande and Kent (1992), in particular the three reversed polarity intervals in the lower Yakataga Formation (Lagoe et al., 1993, fig. 4) with the similar three intervals in Chron $3 \mathrm{n}$ between 4.13 and 4.81 Ma shown by Cande and Kent (1992, fig. 29). The Berggren et al. (1985) time scale used by Lagoe et al. (1993) shows only two reversed intervals within Chron $3 \mathrm{n}$. Such a reinterpretation gives a date of the lowest ice-rafted material in the Yakataga Formation of 4.20-4.27 Ma, the same age as the first occurrence of IRD at Site 887. Using this correlation, we interpret the increased flux of terrigenous material to Site 887 beginning about $4.3 \mathrm{Ma}$ as recording enhanced glaciation in the Gulf of Alaska region, including some alpine glaciers that reached sea level.

Among the important objectives for Site 887 was to determine the glacial history of Alaska as interpreted by both the IRD record (Krissek, this volume) and the record of overall terrigenous deposition given above. The terrigenous flux signal shows an increase in the input of clastics to the ocean beginning at $2.8 \mathrm{Ma}$ and peaking at $2.6 \mathrm{Ma}$, the "accepted" time for the onset of Northern Hemisphere glaciation. The MAR of the coarse fraction (IRD) increases at 2.6 Ma (Fig. 10A; Krissek, this volume). A relative maxima of terrigenous fluxes is centered at about $1.5 \mathrm{Ma}$, ending with a low at $1.1 \mathrm{Ma}$. The last $0.9 \mathrm{~m} . \mathrm{y}$. have been characterized by the greatest terrigenous fluxes, peaking at about 0.6 and $0.25 \mathrm{Ma}$ (Fig. 10). The IRD flux maxima are associated with the overall flux maxima.

Physical erosion of the mountainous regions of Alaska and western Canada may be related to the magnitude and duration of the many glacial advances and retreats. As an indication of the relative magnitude of the individual glacial advances, we have determined the amplitude of the benthic $\delta^{18} \mathrm{O}$ variations over the past $3 \mathrm{~m}$.y. (Fig. 10B). Comparison of the two records, terrigenous fluxes and amplitude of glacial cycles, shows both similarities and differences. The initial increase in the amplitude of the $\delta^{18} \mathrm{O}$ glacial cycles from about 3.0 to 2.6 Ma essentially is matched by the increase in terrigenous fluxes, as is the younger large increase in both cycle amplitude and flux between 1.1 and $0.9 \mathrm{Ma}$. One relative minimum in cycle amplitude at 1.1 Ma matches a flux minimum, but other minima at $1.85 \mathrm{Ma}$ and $0.55 \mathrm{Ma}$ correspond with rising or high terrigenous fluxes (Fig. 10A and B).

It would seem that increases in the scale of glaciation, as recorded by the increases in the amplitude of the oxygen isotope curve, result in increases in the rate and volume of erosion in Alaska. The three relative flux maxima at 2.6, 1.5, and $0.6 \mathrm{Ma}$ all immediately follow increases in the amplitude of glacial-interglacial cycles. Furthermore, fluxes do not remain high if or when cycle amplitude remains high (compare the two plots for the 1.6-1.3 Ma interval and the $0.6-0 \mathrm{Ma}$ interval). This implies that there is a decreasing return on the glacial erosion investment, so that for every successive advance of a given magnitude down a glaciated valley there is less material eroded than during the previous advance. Readily erodible material is removed and only the more resistant bedrock remains, covered perhaps with a thin veneer of regressional moraine. Only when the size (or duration) of the glaciers increases does the erosive ability of the glaciers increase again.

\section{SUMMARY}

Biogenic sediment fluxes in the Gulf of Alaska record both global and regional paleoceanographic events. The worldwide productivity maximum that occurred in the latest Miocene through early to middle Pliocene is seen as a two- to threefold increase in the MAR of opal. A younger Pleistocene maximum in opal flux is confined to the Gulf of Alaska and may reflect an increase in the amount of upwelling associated with the Alaska Gyre. Calcium carbonate occurs occasionally in the sedimentary section with lesser peaks occurring at about 14,10 , and $8 \mathrm{Ma}$. The major episode of calcite deposition coincides in time with the mid-Pliocene warm interval and may reflect changing oceanographic conditions.

Terrigenous fluxes provide a record dominated by continental uplift and glacial erosion. The Gulf of Alaska record at Site 887 allows us to estimate the time of Coast Range uplift and the beginning of the deposition of the Surveyor Fan at $9.7 \mathrm{Ma}$, the possible initiation of 


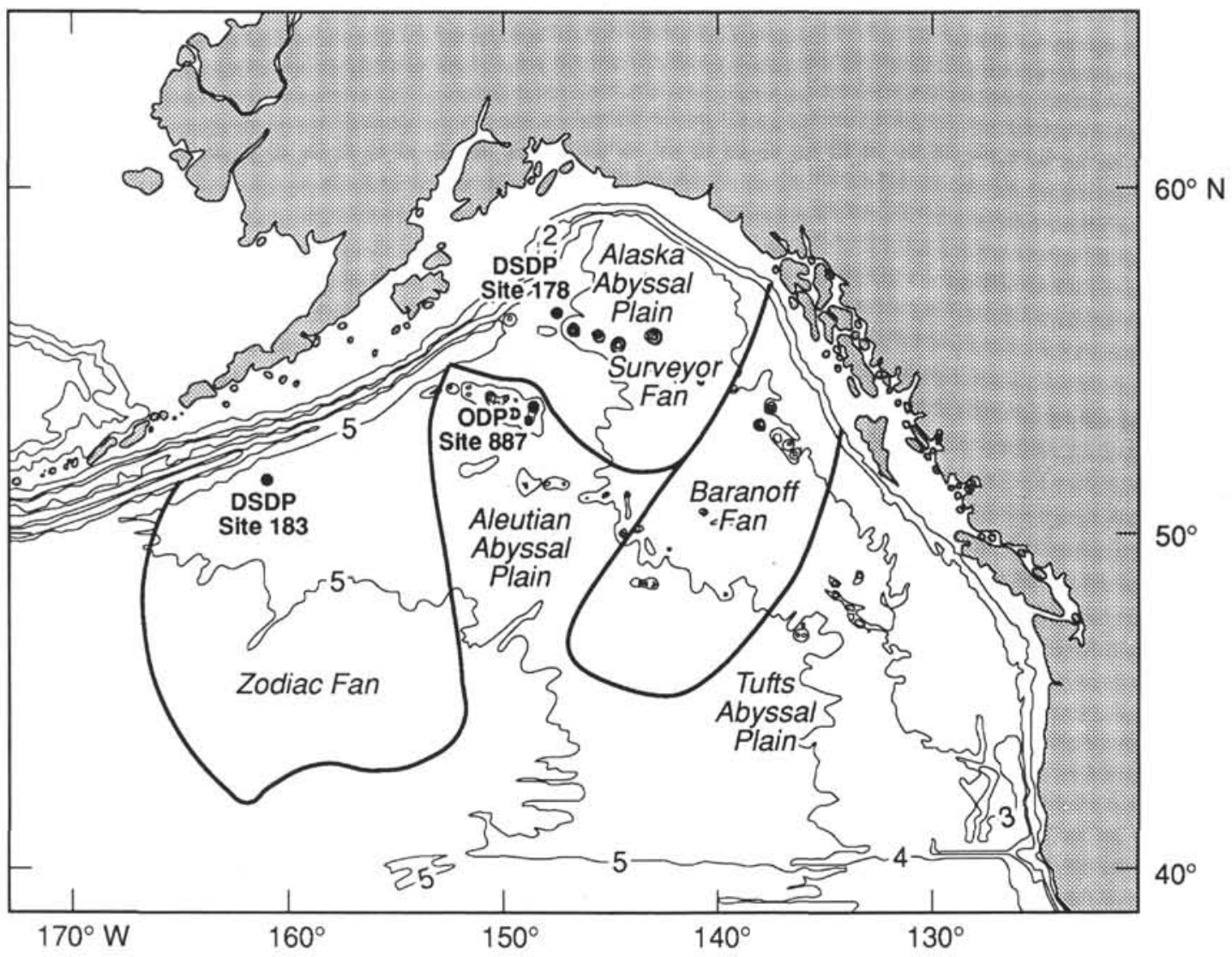

Figure 11. Map showing the deep-sea fans and abyssal plains of the Gulf of Alaska (after von Huene, 1989). Location of ODP Site 887 and DSDP Sites 178 and 183 are shown. Contour intervals in kilometers.

Alpine glaciation and beginning of deposition of the Baranoff Fan at 5.7 Ma, and the first IRD evidence of these glaciers reaching sea level at 4.2 Ma. Deposition associated with the Pliocene-Pleistocene Northern Hemisphere glaciation has its earliest stages beginning about $2.9 \mathrm{Ma}$ and reaches relative maxima at 2.6, 1.5, and $0.6 \mathrm{Ma}$. These terrigenous flux peaks occur in association with increases in both the IRD flux and the amplitude of glacial/interglacial cycles as seen in the benthic $\delta^{18} \mathrm{O}$ record of continental ice volume.

\section{ACKNOWLEDGMENTS}

We thank our colleagues on ODP Leg 145 for an enjoyable cruise and extensive discussion on topics of the depositional history of the North Pacific. This manuscript was reviewed by Mike Underwood and Andy Stevenson whom we thank for useful comments and suggestions. The U.S. Science Support Program of the Joint Oceanographic Institutions provided financial support for this effort.

\section{REFERENCES*}

Backman, J., Duncan, R.A., et al., 1988. Proc. ODP, Init. Repts., 115: College Station, TX (Ocean Drilling Program).

Berggren, W.A., Kent, D.V., Flynn, J.J., and Van Couvering, J.A., 1985. Cenozoic geochronology. Geol. Soc. Am. Bull., 96:1407-1418.

\footnotetext{
"Abbreviations for names of organizations and publications in ODP reference lists follow the style given in Chemical Abstracts Service Source Index (published by American Chemical Society).
}

Cande, S.C., and Kent, D.V., 1992. A new geomagnetic polarity time scale for the Late Cretaceous and Cenozoic. J. Geophys. Res., 97:1391713951.

Clemens, S.C., and Prell, W.L., 1990. Late Pleistocene variability of Arabian Sea summer monsoon winds and continental aridity: eolian records from the lithogenic component of deep-sea sediments. Paleoceanography, 5:109-145.

Creager, J.S., Scholl, D.W., et al., 1973. Init. Repts. DSDP, 19: Washington (U.S. Govt. Printing Office).

Dodimead, A.J., Favorite, F., and Hirano, T., 1963. Salmon of the North Pacific, II. Review of the oceanography of the subarctic Pacific region. Bull. Int. N. Pac. Fisheries Comm., 13.

Hays, J.D. (Ed.), 1970. Geological Investigations of the North Pacific. Mem.-Geol. Soc. Am., 126.

Hovan, S.A., in press. Late Cenozoic atmospheric circulation intensity and climatic history recorded by eolian deposition in the eastern equatorial Pacific Ocean, Leg 138. In Pisias, N.G., Mayer, L.A., Janecek, T.R., Palmer-Julson, A., and van Andel, T.H. (Eds.), Proc. ODP., Sci. Results, 138: College Station, TX (Ocean Drilling Program).

INPAC (International N.E. Pacific Activities Consortium), 1985, The Workshop Report on Drilling in the N.E. Pacific. Seattle, Washington.

Kroenke, L.W., Berger, W.H., Janecek, T.R., et al., 199I. Proc, ODP, Init. Repts., 130: College Station, TX (Ocean Drilling Program).

Kulm, L.D., von Huene, R., et al., 1973. Init. Repts. DSDP, 18: Washington (U.S. Govt. Printing Office).

Lagoe, M.B., Eyles, C.H., Eyles, N., and Hale, C., 1993. Timing of late Cenozoic tidewater glaciation in the far North Pacific. Geol. Soc. Am. Bull., 105:1542-1560.

Mayer, L., Pisias, N., Janecek, T., et al., 1992. Proc. ODP, Init. Repts., 138 (Pts. 1 and 2): College Station, TX (Ocean Drilling Program).

Menard, H.W., 1953. Pleistocene and Recent sediment from the floor of the northeastern Pacific Ocean. Geol. Soc. Am. Bull., 65:1279-1294. 1955. Deep-sea channels, topography and sedimentation. AAPG Bull., 39:236-255, 
1964. Marine Geology of the Pacific: New York (McGraw-Hill).

Moore, T.C., Jr., 1993. The super-productive and super-El Nino oceans. Stratigraphic Record of Global Change. SEPM Meeting Abstr. Program. 54-55. (Abstract)

Moore, T.C., Jr., Rabinowitz, P.D., et al., 1984. Init. Repts. DSDP, 74: Washington (U.S. Govt. Printing Office).

Peterson, L.C., Murray, D.W., Ehrmann,W.U., and Hempel, P., 1992. Cenozoic carbonate accumulation and compensation depth changes in the Indian Ocean. In Duncan, R.A., Rea, D.K., Kidd, R.B., von Rad, U., and Weissel, J.K. (Eds.), Synthesis of Results from Scientific Drilling in the Indian Ocean. Geophys. Monogr., Am. Geophys Union, 70:311-333. [12-9-95]

Raymo, M.E., Hodell, D., and Jansen, E., 1992. Response of deep ocean circulation to initiation of Northern Hemisphere glaciation (3-2 Ma). Paleoceanography, 7:645-672.

Rea, D.K., 1992. Delivery of Himalayan sediment to the northern Indian Ocean and its relation to global climate, sea level, uplift, and seawater strontium. In Duncan, R.A., Rea, D.K., Kidd, R.B., von Rad, U., and Weissel, J.K. (Eds.), Synthesis of Results from Scientific Drilling in the Indian Ocean. Geophys. Monogr., Am. Geophys. Union, 70:387-402. 208-210.

Rea, D.K., Basov, I.A., Janecek, T.R., Palmer-Julson, A., et al., 1993. Proc. ODP, Init. Repts., 145: College Station, TX (Ocean Drilling Program).

Rea, D.K., and Duncan, R.A., 1986. North Pacific Plate convergence: a quantitative record of the past 140 m.y. Geology, 14:373-376.

Rea, D.K., and Janecek, T.R., 1981. Mass-accumulation rates of the nonauthigenic inorganic crystalline (eolian) component of deep-sea sediments from the western mid-Pacific Mountains, Deep Sea Drilling Project Site 463. In Theide, J., Vallier, T.L., et al., Init. Repts. DSDP, 62: Washington (U.S. Govt. Printing Office), 653-659.
Rea, D.K., Pisias, N.G., and Newberry, T., 1991. Late Pleistocene paleoclimatology of the central Equatorial Pacific: flux patterns of biogenic sediments. Paleoceanography, 6:227-244.

Sikes, E.L., Keigwin, L.D., and Curry, W.B., 1991. Pliocene paleoceanography: circulation and oceanographic changes associated with the $2.4 \mathrm{Ma}$ glacial event. Paleoceanography, 6:245-258.

Stevenson, A.J., and Embley, R., 1987. Deep-sea fan bodies, terrigenous turbidite sedimentation and petroleum geology, Gulf of Alaska. In Scholl, D.W., Grantz, A., and Vedder, J.G. (Eds.), Geology and Resource Potential of the Continental Margin of Western North America and Adjacent Ocean Basins-Beaufort Sea to Baja California. Circum-Pac. Counc. Energy Min. Resour., Earth Sci. Ser., 503-522.

von Huene, R., 1989. Continental margins around the Gulf of Alaska. In Winterer, E.L., Hussong, D.M., and Decker, R.W. (Eds.), The Geology of North America (Vol. N): The Eastern Pacific Ocean and Hawaii. Geol. Soc. Am., 383-401.

Woodruff, F., and Savin, S.M., 1989. Miocene deepwater oceanography. Paleoceanography, 4:87-140.

Wright, J.D., Miller, K.G., and Fairbanks, R.G., 1991. Evolution of modern deepwater circulation: evidence from the late Miocene Southern Ocean. Paleoceanography, 6:275-290.

1992. Early and middle Miocene stable isotopes: implications for deepwater circulation and climate. Paleoceanography, 7:357-389.

\author{
Date of initial receipt: 6 April 1994 \\ Date of acceptance: 25 January 1995 \\ Ms 145SR-122
}

